\title{
APLICABILIDADE DO BRUMS: ESTADOS DE HUMOR EM ATLETAS DE VOLEIBOL E TÊNIS NO ALTO RENDIMENTO
}

\author{
APPLICABILITY OF BRUMS: MOOD PROFILE IN ATHLETES OF VOLLEYBALL AND TENNIS \\ IN HIGH PERFORMANCE
}

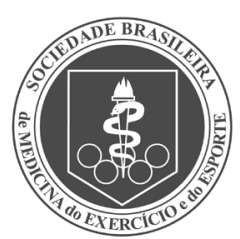

Artigo Original

\section{APLICABILIDAD DE BRUMS: ESTADOS DE HUMOR EN ATLETAS DE VÓLEIBOL Y TENIS DE ALTO DESEMPEÑO}

\begin{abstract}
Tatiana Marcela Rotta' (Psicóloga) Izabel Cristina Provenza de Miranda Rohlfs² (Educadora Física)

Walter Ferreira de Oliveira ${ }^{3}$ (Médico Psiquiatra)

1. Universidade do Sul de Santa Catarina, Florianópolis, SC. Brasil. 2. Minas Tênis Clube, Belo Horizonte, MG, Brasil. 3. Universidade Federal de Santa Catarina, Florianópolis, SC, Brasil.
\end{abstract}

\section{Correspondência:}

Rua das Corticeiras, 1250, Campeche, Florianópolis, SC. Brasil. 88063-160. tatianamarcelar@hotmail.com

\section{RESUMO}

Introdução: Os estados de humor são indicadores que auxiliam sobremaneira o rendimento e a prevenção da saúde do atleta. Objetivo: Analisar a aplicabilidade do instrumento BRUMS na avaliação do perfil de estados de humor em atletas de alto rendimento do sexo masculino, de voleibol $n=59$ e tênis $n=69$. Métodos: comparar as variáveis independentes: modalidade esportiva (voleibol e tênis); tempo de prática no alto rendimento (até 2 anos; mais de 2 anos) e categorias de idade (jovens e adultos) com as variáveis dependente do perfil de humor (tensão, depressão, raiva, vigor, fadiga e confusão mental). O estudo causal-comparativo, utilizou, para coleta de dados, o instrumento BRUMS, validado no Brasil, com participação das autoras desse estudo. Resultados: Ao executar a MANOVA, foram verificadas diferenças entre as modalidades ( $F=4,289 / p=0,001$; Hotellings's Trace $=0,216)$ e tempo de prática $(F=5,845 / p<0,001$; Hotelling's Trace $=0,295)$ no vigor. A modalidade versus tempo de prática apresentou significância na interação das duas variáveis ( $p=0,003$ ), em torno de 7\% da variância. Também na variável tensão $p=0,05$ (voleibol vs. tempo de prática), na variável raiva $p=0,001$ (voleibol vs. categoria de idade). No tênis, a depressão $p=0,001$, raiva $p=0,04$ e confusão mental $p<0,02$ com médias maiores em adultos e mais experientes. Conclusão: A modalidade foi responsável por $11 \%$ da alteração no perfil de humor ( $p=0,001)$.

Palavras-chave: afeto, voleibol, tênis.

\section{ABSTRACT}

Introduction: Mood states are indicators that greatly help the performance and the prevention of health problems in athletes. Objective: To analyze the applicability of BRUMS questionnaire to assess mood states in volleyball $(n=59)$ and tennis $(n=69)$ male high performance athletes. Methods: To compare the independent variables: sport (volleyball and tennis); practice time in high performance (up to 2 years, more than 2 years) and age groups (youth and adults) with the dependent variables of the mood profile (tension, depression, anger, vigor, fatigue and confusion). The causal-comparative study used for data collection, BRUMS questionnaire, was validated in Brazil with the participation of the author of this study. Results: When performing the MANOVA, differences were found between the sports ( $F=4.289 / p=0.001$; Hotelling's Trace $=0.216)$ and practice time ( $F=5.845 / p<0.001$, Hotelling's Trace $=0.295)$ in force. The sport compared with practice time showed a significant interaction of the two variables $(p=0.003)$ of approximately $7 \%$ of the variance. This was also demonstrated in the variables tension ( $p=0.05$ - volleyball vs. practice time), anger ( $p=0.001$-volleyball vs. age category). In tennis, depression $p=0.001$, anger $p=0.04$, confusion $p<0.02$ with larger average in adults more experienced. Conclusion: The mode was responsible for $11 \%$ of the change in mood profile $(p=0.001)$.

Keywords: affect, volleyball, tennis.

\section{RESUMEN}

Introducción: Los estados de humor son indicadores que auxilian sobremanera al rendimiento y a la prevención de la salud del atleta. Objetivo: Analizar la aplicabilidad del instrumento BRUMS en la evaluación del perfil de estados de humor en atletas de alto rendimiento del sexo masculino, de vóleibol $n=59$ y tenis $n=69$. Métodos: comparar las variables independientes: modalidad deportiva (vóleibol y tenis); tiempo de práctica en alto rendimiento (hasta 2 años; más de 2 años) y categorías de edad (jóvenes y adultos) con las variables dependientes del perfil de humor (tensión, depresión, resentimiento, vigor, fatiga y confusión mental). El estudio causal-comparativo, usó, para colecta de datos, el instrumento BRUMS, validado en Brasil, con participación de la autora de este estudio. Resultados: Al ejecutar MANOVA, fueron verificadas diferencias entre las modalidades ( $F=4,289 / p=0,001$; Hotelling's Trace $=0,216)$ y tiempo de práctica ( $F=5,845 / p<0,001$; Hotelling's Trace $=0,295)$ en el vigor. La modalidad versus tiempo de práctica presentó significación en la interacción de las dos variables 
( $p=0,003$ ), alrededor de 7\% de variación. También en la variable tensión $p=0,05$ (vóleibo/ vs. tiempo de práctica), en la variable resentimiento $p=0,001$ (vóleibol vs. categoría de edad). En el tenis, la depresión $p=0,001$, resentimiento $p=0,04$ y confusión mental $p<0,02$ con promedios mayores en adultos y con más experiencia. Conclusión: La modalidad fue responsable por $11 \%$ de la alteración en el perfil de humor $(p=0,001)$.

Palabras clave: afecto, vóleibol, tenis.

\section{INTRODUÇÃO}

A associação entre a emoção e o desempenho esportivo surgiu quando um grupo de pesquisadores se interessou pelas variáveis dependentes da performance (área motora), enquanto outro grupo concentrou-se nas variáveis independentes que influenciam a performance (os aspectos emocionais). A área de aprendizagem motora e de controle motor aproximou-se dos estudos da psicologia no que se refere aos processos cognitivos e neuropsicológicos envolvidos no movimento. ${ }^{1}$

Mas, como mensurar tais emoções em atletas para o desempenho esportivo? Alguns instrumentos são conhecidos para mensurar os estados de humor compostos pelos fatores de tensão, depressão, raiva, vigor, fadiga, confusão mental, sendo o vigor inversamente proporcional na leitura dos fatores avaliados. O Profile Mood States (POMS) foi criado para utilização psiquiatra em 1971 por Macnair, Lorr e Dropleman², e validado para ser utilizado no contexto esportivo por Morgan, com 64 itens avaliando estados de humor (tensão, depressão, raiva, vigor, fadiga e confusão mental). No Brasil, o instrumento POMS foi validado para uso em atletas na tese de doutorado de Brandão ${ }^{4}$. Outras versões desse instrumento foram, validadas em formato reduzido ${ }^{5}$,em atletas de Portugal, e também o POMS A, validado para atletas jovens, menores de 18 anos, para língua inglesa ${ }^{6}$.

A partir de estudos na relação dos estados de humor e performance esportiva, derivado do POMS A ${ }^{6}$, foi criado para utilização em atletas de língua inglesa, jovens e adultos ${ }^{7}$ o Brunel Mood States (BRUMS). Também proposto a partir da validação para população não atleta, em duas categorias de idade, jovem e adulto. O BRUMS no Brasil foi traduzido e adaptado para uso de atletas e não atletas ${ }^{8-10}$.

O objetivo do estudo foi avaliar a aplicabilidade do instrumento BRUMS a partir da comparação das variáveis dependentes: estados de humor (tensão, depressão, raiva, vigor, fadiga e confusão mental) e variáveis independentes: a) modalidades esportivas (individual (tênis) e coletivas (voleibol); b) tempo de experiência (até 2 anos; mais de 2 anos); c) categorias de idade (atletas jovens e adultos).

\section{MÉTODOS}

Esta pesquisa caracteriza-se como causal-comparativa, pois visa estabelecer uma correspondência das razões ou causas para o estado atual do fenômeno que permeia o estudo em atletas de alto rendimento: variáveis dependentes: estados de humor (tensão, depressão, raiva, vigor, fadiga e confusão mental); variáveis independentes, a) modalidades esportivas (individual (tênis) e coletivas (voleibol); b) tempo de experiência (até 2 anos; mais de 2 anos); c) categorias de idade (atletas jovens e adultos). ${ }^{11}$

O número de participantes do estudo na modalidade de voleibol foram 59 atletas e no tênis 69, totalizando 128 atletas, todos do sexo masculino. Os participantes possuem características extremas de uma variável, por exemplo, ser atleta jovem (15 a 18 anos) ou adulto (> 18 anos), de esportes de modalidade esportiva (coletiva= voleibol e individual=tênis de campo), possuir treinamento esportivo e experiência na prática esportiva regular, em competições de níveis nacionais e internacionais (alto rendimento). A idade mínima dos atletas de voleibol é de 15 anos e a máxima de 28 (média= 19,02) $\mathrm{s}=3,516$ ), já no tênis a média de idade foi menor (média=17,01/ $\mathrm{s}=2,09)$, variando de 15 a 23 anos de idade. Ao comparar o número de atletas no voleibol e no tênis, divididos por categoria de idade, não foi verificada homogeneidade entre os grupos, sendo 23 atletas jovens e 36 adultos no voleibol, enquanto no tênis tem-se 55 atletas jovens e 14 adultos.

Na variável tempo de prática dos atletas, os participantes foram divididos em dois níveis: 1) até 2 anos de prática; 2) mais de 2 anos de prática, no alto rendimento. Verificou-se idade média de 16,43 $(s=1,003 /-15+16)$ anos para o nível 1 , e de 20,46 $(s=3,494 /-17+28)$ para o nível 2, no grupo total de participantes. Ao comparar as médias de idade dos participantes com até 2 anos de experiência com aqueles com mais de 2 anos, foram verificadas diferenças entre os grupos $(t=-7,806 / p<0,001)$, indicando que o grupo com menor tempo de experiência é o que tem média de idade significativamente inferior. Quando o número de participantes de cada modalidade esportiva é divido por tempo de experiência, verifica-se uma amostra homogênea na comparação do voleibol e tênis: 38 atletas com até dois anos de experiência e 21 com mais de 2 anos de experiência no voleibol; 42 atletas com até 2 anos de experiência e 27 atletas com mais de 2 anos de experiência no tênis.

O instrumento utilizado foi a escala BRUMS, validado no Brasil ${ }^{8-10}$. Após aprovação do Comitê de Ética da Universidade do Estado de Santa Catarina (UDESC), contatou-se os pesquisados e responsáveis quando menores de 18 anos para iniciar o estudo. Após autorização, apresentou-se o Termo Livre e Consentimento Esclarecido com recoIhimento da assinatura dos mesmos.

Foi realizada a aplicação da escala BRUMS, nos contextos esportivos dos atletas pesquisados. O BRUMS levou cerca de 1 a 5 minutos para ser respondido por cada pesquisado. Os pesquisados foram inquiridos conforme o enunciado da escala "Como você se sente agora?", embora outras formas "Como você tem se sentido nesta última semana, inclusive hoje?", ou "Como você normalmente se sente?" possam ser usadas.

O instrumento BRUMS avalia estado de humor de atletas e não atletas, jovens e adultos, possuindo 24 itens no total sendo divididos em seis itens representativos para cada fator de estados de humor que são: fadiga, depressão, raiva, vigor, confusão mental, tensão. Os atletas optam por atribuir pontos para cada item que representa os estados de humor : ( 0 = absolutamente não; $1=$ um pouco; 2 = moderadamente; $3=$ bastante; $4=$ extremamente). Os escores brutos são equivalentes e padronizados ( $T$ escore), ilustrado para cada campo e convertido em escore padrão de 50 (média do percentil 50). Há distintos escores padrões para atletas adultos e jovens, que são representados de forma descritiva no gráfico (figura 1 e 2$)^{7-9}$.

Para verificar possíveis efeitos interativos entre as variáveis foi utilizado para análise dos dados, a análise multivariada de variância (MANOVA), com desenho: seis (estados de humor) versus duas (modalidade) versus duas (tempo de prática) ${ }^{11}$. Considerando que as 
variáveis são intervalares, a análise estatística foi realizada com base em testes paramétricos, observando, primeiramente, a normalidade da distribuição por meio de histogramas, homogeneidade de variâncias, média, desvio-padrão, que são pré-requisitos para a realização de testes paramétricos ${ }^{11}$. Também utilizou-se o Box's M Test ${ }^{12}$ para verificar se houve ou não violação na homogeneidade das matrizes de variância-covariância. O teste utilizado para o cálculo do valor F foi o Hotelling's Trace, devido à característica bivariada de cada variável independente ${ }^{11}$.

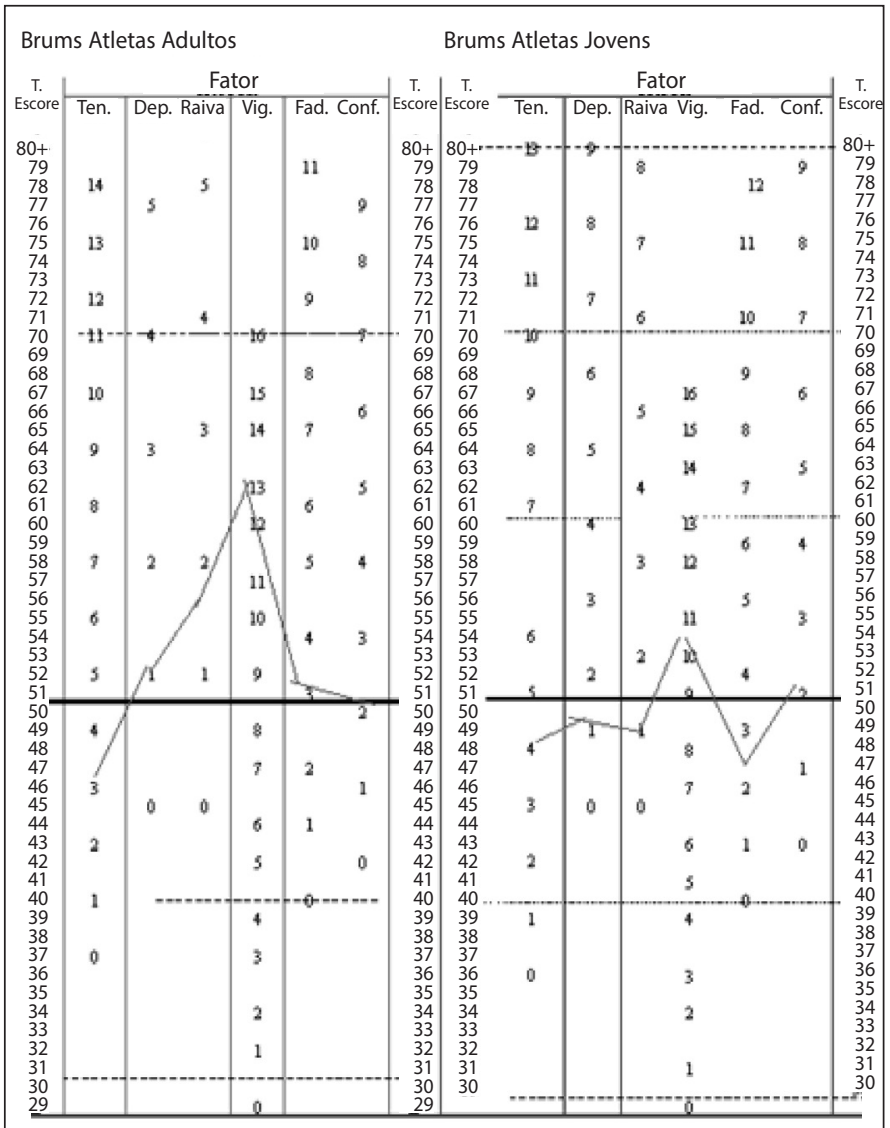

Manual do Brums no Brasil10,11.

Figura 1. Escore padrão do BRUMS: atletas adultos e jovens para modalidade de voleibol.

\section{RESULTADOS}

Foram analisados os dados pesquisados a partir do desenho estatístico utilizado. Ao analisar o Box's M test, não foi verifica igualdade nas matrizes de covariância. Contudo, ao executar a MANOVA, a análise multivariada de variância, foram verificadas diferenças multivariadas entre as modalidades ( $F=4,289 / p=0,001$; Hotellings's Trace $=0,216)$ e tempo de prática $(F=5,845 / p<0,001$; Hotelling's Trace $=0,295$ ). Além disso, foram verificados efeitos interativos da modalidade e tempo de prática na variável independente (estados de humor) ( $F=2,943 / p=0,01$; Hotelling's Trace $=0,148)$. Os valores de $\eta^{2}$ parcial sugerem que a variável modalidade foi responsável por 18\% da variação na combinação linear das variáveis dependentes (estados de humor), enquanto que o tempo de prática respondeu por $23 \%$ dessa variação. A interação da modalidade com o tempo de prática foi responsável por 13\% da variação da combinação linear dos estados de humor.

A tabela 1 foi elaborada para demonstrar o quanto cada estado de humor varia em função das modalidades, do tempo de prática e da

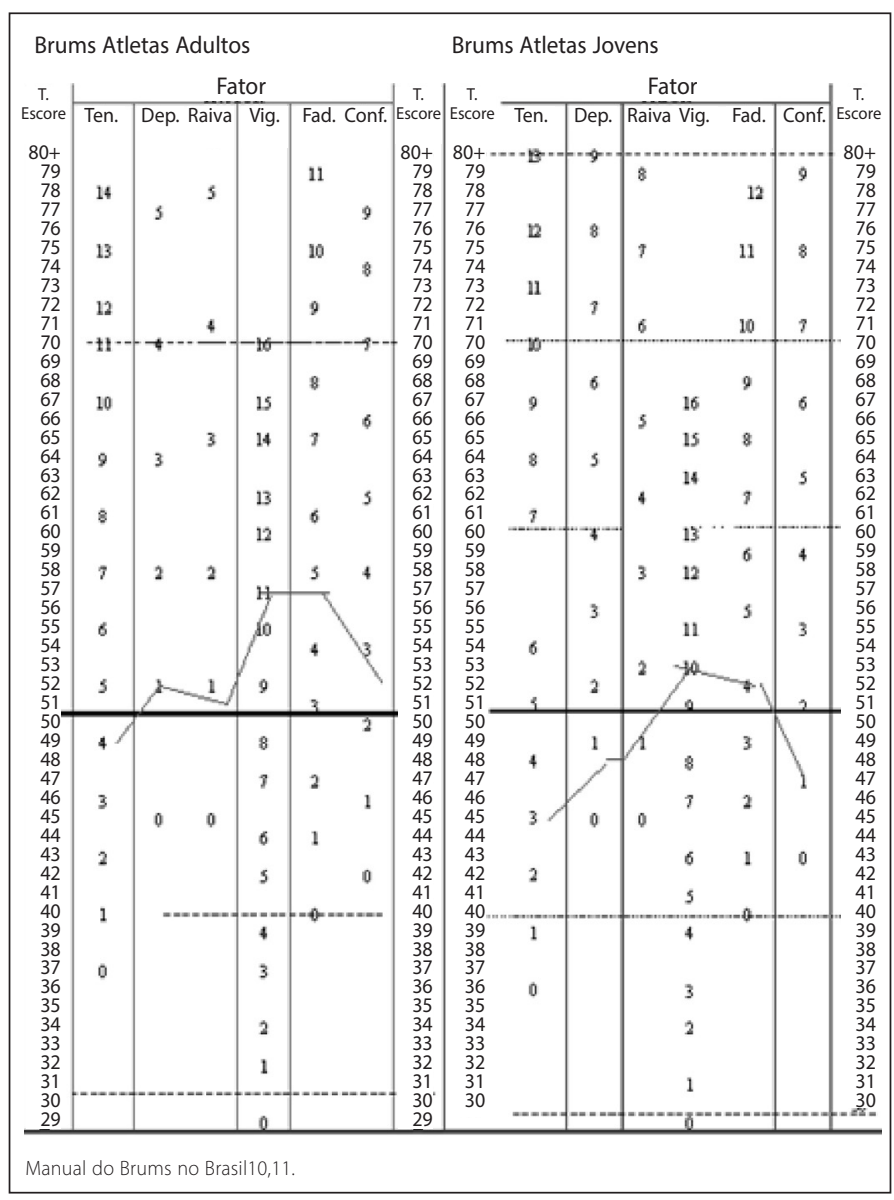

Figura 2. Escore padrão do BRUMS atletas adultos e jovens para modalidade de tênis,

interação entre esses, ou seja, os efeitos principais. Na Tabela 1 consta as variáveis independentes e dependentes separadamente, bem como homogeneidade de variância (F), a significância (p) e o partial eta squared (partial $\eta^{2}$ ) para análise do tamanho de efeito.

Ao analisar os efeitos separados por variável, apresentados na tabela 1, observa-se poucas diferenças nos estados de humor individualmente. Contudo, os resultados da MANOVA em relação a variável independente modalidade esportiva $X$ a variável dependente estados de humor, demonstrou diferença significativa na variação do vigor, $F=15,647, p<0,001, \eta^{2}$ parcial 0,112. Da mesma forma, na variável independente tempo de prática $X$ variável estados de humor, há influência no resultado, $F=14,976, p<0,001, \eta^{2}$ parcial 0,108. Os valores de parcial $\eta^{2}$ sugerem que a modalidade esportiva e o tempo de prática foram responsáveis por $11 \%$ da variação na combinação linear da variável dependente (vigor). Além disso, foram encontrados efeitos interativos, a partir da análise de variância $2 \times 2$ da modalidade versus tempo de prática modalidade, para a confusão mental, F=9,179, $p<0,003, \eta^{2}$ parcial 0,069).

Os efeitos interativos da modalidade e tempo de prática na confusão mental, apresentados nos resultados da tabela 1, $p=0,003$, foram comparadas as médias dos estados de humor, em função de cada modalidade e tempo de prática (figura 3).

Os atletas com mais de dois anos de prática no alto rendimento apresentaram níveis semelhantes de confusão mental, enquanto que os atletas com até dois anos de prática no voleibol apresentaram índices superiores de confusão mental, sem nenhuma sobreposição de escores (figura 2). Assim, o nível de confusão mental dos menos experientes foi dependente da modalidade.

As figuras 1 e 2, representam os gráficos da escala de BRUMS a partir dos resultados provenientes do escore padrão dos estados de humor 
Tabela 1. Diferenças e efeitos principais da modalidade, tempo de prática e interação nos estados de humor.

\begin{tabular}{|c|c|c|c|c|}
\hline Variável Independente & Estados de Humor & $F$ & $\mathrm{p}$ & $\eta^{2}$ partial. \\
\hline & Tensão & 0,40 & 0,842 & 0,000 \\
\hline & Depressão & 3,754 & 0,55 & 0,029 \\
\hline \multirow[t]{6}{*}{ Modalidade } & Raiva & 4,065 & 0,046 & 0,032 \\
\hline & Vigor & 15,647 & $<0,001^{*}$ & 0,112 \\
\hline & Fadiga & 4,945 & 0,028 & 0,038 \\
\hline & Confusão Mental & 0,438 & 0,509 & 0,004 \\
\hline & Tensão & 1,748 & 0,189 & 0,014 \\
\hline & Depressão & 0,296 & 0,587 & 0,002 \\
\hline \multirow[t]{6}{*}{ Tempo de Prática } & Raiva & 2,685 & 0,104 & 0,021 \\
\hline & Vigor & 14,976 & $<0,001^{*}$ & 0,108 \\
\hline & Fadiga & 1,459 & 0,229 & 0,012 \\
\hline & Confusão mental & 0,013 & 0,908 & 0,000 \\
\hline & Tensão & 4,212 & 0,042 & 0,033 \\
\hline & Depressão & 5,258 & 0,024 & 0,041 \\
\hline \multirow[t]{4}{*}{ Interação } & Raiva & 0,022 & 0,882 & 0,000 \\
\hline & Vigor & 2,512 & 0,116 & 0,020 \\
\hline & Fadiga & 1,215 & 0,273 & 0,010 \\
\hline & Confusão mental & 9,179 & $0,003^{*}$ & 0,069 \\
\hline
\end{tabular}

* Diferença significativa ao nível de $p<0,0125$

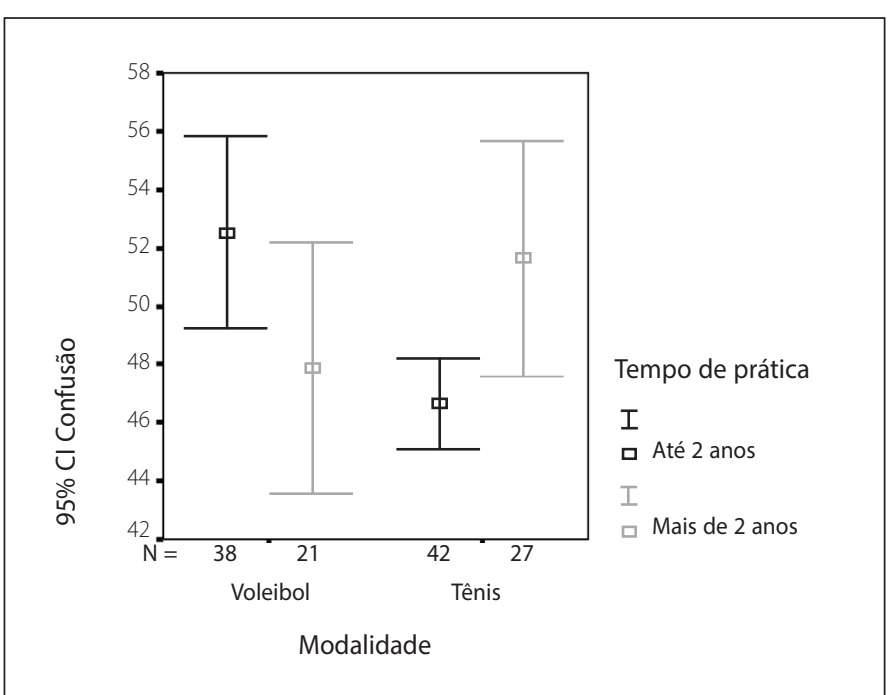

Figura 3. Intervalos de confiança: modalidade (voleibol e tênis) e o tempo de prática (até dois anos e mais de dois anos ) na confusão mental.

(tensão, depressão, raiva, vigor, fadiga, confusão mental) , nessa ordem da esquerda para direita. Dessa forma, representada de forma descrita nos gráficos padrões, o fator tensão a partir das médias dos escores do BRUMS em ambas modalidades esportivas e categorias de idade. 0 fator depressão nos atletas adultos de voleibol e tênis as médias foram equivalentes. Já no fator raiva, foram os atletas adultos de voleibol que resultaram em médias maiores do que os adultos tenistas adultos, o que não foi verificado entre os atletas jovens. Ainda, o fator vigor expressa médias maiores na modalidade de voleibol jovem e adulto quando relacionado aos atletas de tênis também jovens e adultos. Em contrapartida, os atletas de tênis adultos e jovens demonstram médias maiores no fator fadiga do que o grupo de atletas de voleibol adultos e jovens. E, o fator confusão mental, difere-se sendo que nos atletas adultos de tênis as médias foram maiores do que os atletas adultos de voleibol, e de forma inversa, médias maiores em atletas jovens de voleibol do que atletas de tênis jovem.

\section{DISCUSSÃO}

Em relação aos dados descritivos a partir dos escores da escala de BRUMS (figura 1,2), destaque para em ambas as modalidades esportivas (voleibol e tênis) e ambas categorias de idade (jovem e adulto), o fator vigor, sobrepondo à fadiga mental e física, sendo assim, os resultados ajudam a compreender como os sentimentos e a energia psicológica otimizam o desempenho esportivo ${ }^{12-15}$.

O fator confusão mental, indicou na interação entre as modalidades diferenças significativas, (tabela 1 e figura 3) pode ter relação com outras variáveis nos estados de humor, como resposta/resultado de tensão (ansiedade) e à depressão. Sentimentos de incerteza, instabilidade para controle de emoções e atenção ${ }^{16,17}$. Quanto as diferenças significativas, esse aspecto corrobora quanto à necessidade de investigarmos os atletas em sua individualidade, em que alguns atletas podem ter apresentado maiores alterações de estados de humor, dentro do mesmo grupo do desenho estatístico 13,14,18,19.

Ainda, em estudo de revisão observa-se que o perfil de humor com instabilidade emocional independe do sexo, modalidade e idade. Somente nos treinos de alta intensidade observou-se um efeito negativo sobre os estados de humor; o beneficio psicológico do indivíduo está na dependência de uma interação ótima entre o atleta, as características do exercício, o desempenho no esporte e o ambiente ${ }^{20-23}$.

A preocupação nesse estudo visou ao alto rendimento e ao fator tempo de experiência, como variáveis distintas. Estudos apontam que a iniciação em alto rendimento precocemente pode acarretar, entre vários problemas, devido à atividade física intensa a qual se submetem, esgotamento físico, mental e psicológico, interferindo diretamente no desempenho esportivo, principalmente em esportes individuais ${ }^{24-27}$.

Em relação ao objetivo do estudo, quanto a aplicabilidade do instrumento BRUMS, diante da especificidade de fenômenos psicológicos diretamente influenciando a performance de atletas de alto-rendimento, é relevante o estudo para instrumentalizar profissionais de psicologia do esporte. O instrumento BRUMS pode auxiliar o profissional de psicologia no planejamento da preparação psicológica no esporte que atua, e, na contribuição de informações específicas a outros profissionais das ciências do esporte. Os estados de humor são apontados pela literatura como parâmetros fidedignos de prevenção da saúde do atleta e na maximização da performance dos mesmos, quando comparados com indicadores fisiológicos de cargas físicas e recuperação dos mesmos.

Dessa forma, como limitação do estudo, os estados emocionais de humor são fenômenos psicológicos, e consequentemente fator oscilatório e relacionado com a subjetividade do próprio avaliado, ratifica-se que haverá alterações nos estado do humor de forma individualizada. Para tanto a medida BRUMS de avaliações de estados de humor demonstrou-se sensibilidade para ser utilizada nos acompanhamentos dos estados emocionais relacionados com a prática esportiva. 


\section{CONCLUSÃO}

Desse modo, considera-se relevante a necessidade do acompanhamento, aplicando o instrumento BRUMS para delinear os perfis de estados de humor em atletas de alto rendimento, em momentos distintos do calendário esportivo, e, existe também a necessidade de cuidar com a variabilidade do delineamento da curva do gráfico padrão, levando em conta os processos contextuais ao longo do treinamento esportivo.

Destaque aqui, a aplicabilidade do BRUMS como instrumento de avaliação em Psicologia do Esporte, contexto que carece de material como ferramenta profissional adaptada à realidade esportiva, seja em atletas jovens e adultos, e, de modalidades coletivas e individuais.

\section{AGRADECIMENTOS}

In Memorian - Ao prof. Ruy Jornada Krebs pela dedicação em ensinar, e que participou na construção e orientação desse trabalho. Professor da Pós Graduação de Ciências do Movimento Humano Universidade Estadual de Santa Catarina (UDESC), Brasil.

Todos os autores declararam não haver qualquer potencial conflito de interesses referente a este artigo.

\section{REFERÊNCIAS}

1. De Rose Júnior D. O esporte e a psicologia: enfoque profissional do esporte. In: Rubio K, org. Psicologia do esporte: interfaces, pesquisa e intervenção. São Paulo: Casa do Psicólogo; 2000. p. 29-39.

2. Mcnair DM, Lorr M, Droppleman, LF. Manual for the profile of mood states. San Diego: Educational and Industrial Testing Services; 1971.

3. Morgan WP. Test of champions: the iceberg profile. Psychol Today. 1980;14(2):92-9.

4. Brandão, MR. Fatores de stress em jogadores de futebol profissional [tese]. Campinas, SP: Univer sidade estadual de Campinas; 2000. Disponível em: http://www.bibliotecadigital.unicamp.br/ document/?code=vtls000215630

5. Viana MF, Almeida PL, Santos RC. Adaptação portuguesa da versão reduzida do Perfil de Estados de Humor - POMS. Análise Psicológica; 2001;19(1): 77-92. Disponível em: http://www.scielo.oces.mctes. pt/pdf/aps/v19n1/v19n1a08

6. Terry PC, Lane AM, Lane HJ, Keohane L. Development and validation of a mood measure for adolescents. J Sports Sci. 1999 Nov;17(11):861-72. Disponível em: http://core.kmi.open.ac.uk/download/ pdf/11036776.pdf

7. Terry PC, Lane AM, Fogarty GJ. Construct validity of the. Profile of Mood States-A for use with ad ults. Psychol Sport Exerc. 2003;4:125-39. Disponível em: http://www.nsc.gov.my/media/2014/07/ CJJan2014.pdf

8. Rohlfs IPM, RottaTM, Luft CDB, Carvalho T, Krebs RJ, Andrade A. A escala de humor de Brunel (BRUMS): instrumento para detecção precoce da síndrome do excesso de treinamento Rev Bras Med Esporte. 2008;14(3):176-81.

9. Rotta TM. Perfil de estados de humor em atletas de voleibol e tênis de alto rendimento [dissertação] Florianópolis: Universidade Estadual de Santa Catarina; 2006. Dsiponível em: http://www.tede.udesc br/tde_busca/arquivo.php?codArquivo=1027

10. Appolinário F. Metodologia da ciência: filosofia e prática da pesquisa. São Paulo: Pioneira Thomson Learning; 2006

11. Reis E. Estatística multivariada aplicada. 2a. ed. São Paulo: Silabo; 2001.

12. Weinberg RS, Gould D. Fundamentos da psicologia do esporte e do exercício. Tradução de Maria Cristina Morateiro. 2a. ed. Porto Alegre: Artmed; 2001.

13. O'Connor PJ. Mental energy: Assessing the mood dimension. Athens Nutr Rev. 2006;64(7 Pt 2): S7-9. Disponível em: http://www.researchgate.net/publication/6877755_Mental_energy_Assessing_the_mood_dimension

14. Brandão MR. Perfil psicológico: uma proposta para avaliar atletas. Rev Bras Ciênc Mov. 1993;7(2):16-27. Disponível em: http://portalrevistas.ucb.br/index.php/RBCM/article/view/244
15. BeckAT, Clark DA. Anxiety and depression: an information processing perspective. Anxiety Res. 1988; 1 (1):23 56. Disponível em: http://www.tandfonline.com/doi/abs/10.1080/10615808808248218? journalCode=gasc19\#.VFTI_fnF-So

16. Lane AM, Lovejoy DJ. The effects of exercise on mood changes: the moderating effect of depressed mood. J Sports Med Phys Fitness. 2001;41(4):539-45. Disponível em: https://ulib.derby.ac.uk/ecdu/ CourseRes/dbs/currissu/Lane_A.pdf

17. Brandão MR. Equipe nacional de voleibol masculino: um perfil sócio-psicológico à luz da ecologia do desenvolvimento humano [dissertação]. Santa Maria, RS: Universidade Federal de Santa Maria; 1996.

18. Lázaro JP, Casimiro ESO, Fernandes HMG. Determinação do perfil psicológico de prestação do jogador de handebol português: um estudo em atletas da Liga e da Divisão de Elite.Alto Douro, Portugal.; 2005. [Acesso em 2005 ago. 10] Disponível em: http://www.psicologia.pt/artigos/ textos/A0269.pdf.

19. Brandão, M. R. Perfil psicológico: uma proposta para avaliar atletas. Rev Bras Ciênc Mov 1993;7(2):16-27

20. Aadahl M, Jorgensen T. Validation of a new self-report instrument for measuring physical activity. Med Sci Sports Exerc. 2003;35(7):1196-202.

21. Wong EH, Lox CL, Clark SE. Relation between sports context, competitive trait anxiety, perceived ability, and self-presentation confidence. Percept Mot Skills. 1993;76(3 Pt 1):847-50.

22. Werneck FZ, Bara MG, Ribeiro LC. Efeitos do exercício físico sobre os estados de humor: uma revisão. Rev Bras Psicol Esp Exerc. 2006;1:22-54

23. Spielberger CD. Manual for the State-Trait Anger-expression Inventory. Odessa, FL: Psychologica Assessment Resources; 1991

24. Hanton S, Jones G, Mullen R. Intensity and direction of competitive state anxiety as interpreted by rugby players and rifle shooters. Percept Mot Skills. 2000;90(2):513-21.

25. Lane A, Whyte G, Terry P, Nevill AM. Mood, self-set goals and examination performance: the moderating effect of depressed mood. Personality and Individual Differences. 2005;39:143-53. Disponível em: http://www.sciencedirect.com/science/article/pii/S0191886904003836.

26. Morgan WP. Select psychological factors limiting performance: a mental health model. In: Clarke DH Eckert HM, editors. Limits of human performance. Champaign, IL: Human Kinetics; 1985.

27. Morgan WP, Costill DL. Selected psychological characteristics and health behaviors of aging marathon runners: longitudinal study. Int J Sports Med. 1996;17(4): 305-12. 